\section{CLL verschlechtert Prognose bei Zweittumoren}

Eine chronische lymphatische Leukämie (CLL) erhöht das Risiko für die Entwicklung anderer Krebsentitäten. Beeinflusst die Primärerkrankung CLL auch den natürlichen Verlauf der Zweitmalignitäten? $\mathrm{n}$ einer Überlebensanalyse wurde der Einfluss einer vorbestehenden CLL auf den Krankheitsverlauf verschiedener Sekundärkarzinome untersucht. Dazu wurden für alle Patienten mit Tumoren der Brust ( $\mathrm{n}=579.164)$, von Kolon/Rektum (CRC, $n=412.366)$, Prostata $(n=631.616)$, Lunge $(\mathrm{n}=489.053)$, Niere $(\mathrm{n}=95.795)$,
Pankreas $(\mathrm{n}=82.116)$ und Ovarien $(\mathrm{n}=$ 61.937) aus dem SEER-Programm die Gesamtüberlebensraten abhängig von einer vorbestehenden CLL errechnet.

Patienten mit Karzinomen der Brust (Hazard Ratio [HR] 1,70; p < 0,001), CRC (HR 1,65; p < 0,001), Niere (HR $1,54 ; \mathrm{p}<0,001)$, Prostata (HR 1,92; $\mathrm{p}<$

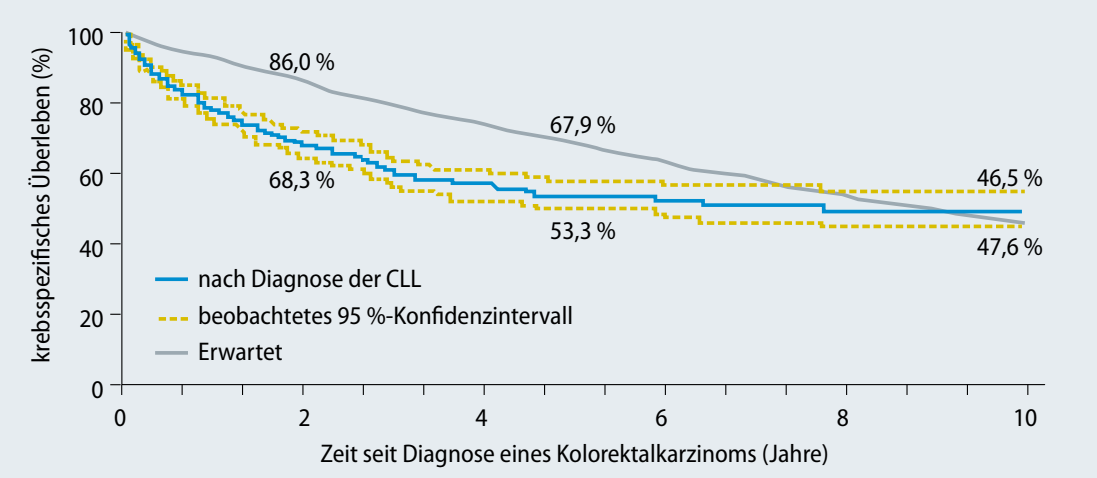

Abb.: Das krebsspezifische Überleben z. B. bei CRC wird durch eine CLL negativ beeinflusst.

\section{RANKL bei multiplem Myelom und CLL}

RANK und sein Ligand spielen eine Schlüsselrolle im Knochenremodelling. Seine Expression, Funktion und therapeutische Beeinflussbarkeit speziell bei malignen Knochendestruktionen, z. B. beim multiplen Myelom, wurden jetzt näher untersucht.

$\mathrm{R}_{\mathrm{k}}^{\mathrm{A}}$ ANKL beeinflusst nicht nur den Knochenstoffwechsel, es spielt auch eine wichtige Rolle in der Pathophysiologie des multiplen Myeloms (MM) und der chronischen lymphatischen Leukämie (CLL). In vitro war bei peripheren mononukleären Zellen von MM und CLL eine deutliche Steigerung der RANKL-Expression auf der Zelloberfläche nachweisbar. Eine Freisetzung von löslichem RANKL fand sich dagegen nur bei MM-Zellen und unterlag einer deutlichen posttranskriptionalen/ posttranslationalen Regulierung. Der RANKL-Signalweg in die MM-Zellen bzw. CLL-Zellen induzierte die FreisetIL-8, die alle am pathophysiologischen Geschehen beteiligt sind.

Die RANKL-Effekte auf Osteoklasten-Genese und Zytokin-Produktion durch die malignen Zellen lassen sich durch die Unterbrechung der RANKRANKL-Interaktion stoppen, z. B. mit dem monoklonalen Antikörper Denosumab. Allerdings ist Denosumab nicht in der Lage, eine immunologische Zerstörung der malignen Zellen herbeizuführen. Die Tübinger Arbeitsgruppe entwickelte deshalb ein RANK-Fc-Fusionsprotein mit modifizierten Fc-Teilen, das eine Zweifachwirkung in Form einer
0,001) oder Lunge (HR 1,19; p < 0,001) hatten nach Adjustierung für Alter, Geschlecht, Ethnie und Krankheitsstadium bei bereits existierender CLL eine verringerte Lebenserwartung. Bei Patienten mit Karzinomen der Brust, CRC und Prostata bleiben diese Zusammenhänge nach Berücksichtigung CLL-induzierter Todesfälle weiterhin signifikant. Auch in diesen Berechnungen war das krebsspezifische Überleben bei Patienten mit präexistenter CLL und sekundären Karzinomen der Brust (HR 1,41; $\mathrm{p}=0,005)$ und CRC (HR 1,46; $<<0,001)$ unabhängig von Alter, Geschlecht, Ethnie und Krankheitsstadium schlechter.

Fazit: Patienten mit CLL, bei denen sich eine zweite Krebserkrankung entwickelt, haben meist eine verkürzte Gesamtüberlebenszeit, v.a. bei Auftreten von Brustkrebs und CRC. Offen sind Fragen nach dem optimalen Behandlungsablauf in dieser Situation oder aggressiveren Screeningprogrammen.

Barbara Kreutzkamp

Solomon BM et al. Overall and cancer-specific survival of patients with breast, colon, kidney, and lung cancers with and without chronic lymphocytic leukemia: A SEER population-based study. J Clin Oncol. 2013;31(7):930-7.

RANKL-Neutralisierung und einer Stimulation der Antikörper-abhängigen zellulären Toxizität natürlicher Killerzellen (NK-Zellen) zeigt. Dieses Fusionsprotein neutralisierte in vitro die RANKL-Effekte auf die Osteoklasten-Genese ähnlich wie Denosumab und stimulierte zusätzlich die NK-Zell-Reaktivität gegen RANKL-exprimierende maligne BZellen. Leztere Eigenschaft war abhängig von der CD16-Affinität des Moleküls.

Fazit: Mithilfe eines Fc-optimierten RANK-Ig-Fusionsproteins können sowohl pathophysiologisch relevante Effekte von RANKL bei MM und CLL blockiert als auch die NK-Antitumorimmunität stimuliert werden. Nun sollte in Invivo-Studien die klinische Effektivität überprüft werden. Barbara Kreutzkamp

Schmiedel BJ et al. RANKL expression, function, and therapeutic targeting in multiple myeloma and chronic lymphocytic leukemia. Cancer Res. 2013;73(2):683-94. 